\title{
A sparse Laplacian in tensor product wavelet coordinates
}

\author{
Tammo Jan Dijkema • Rob Stevenson
}

Received: 27 April 2009 / Revised: 19 December 2009 / Published online: 6 February 2010

(C) The Author(s) 2010. This article is published with open access at Springerlink.com

\begin{abstract}
We construct a wavelet basis on the unit interval with respect to which both the (infinite) mass and stiffness matrix corresponding to the one-dimensional Laplacian are (truly) sparse and boundedly invertible. As a consequence, the (infinite) stiffness matrix corresponding to the Laplacian on the $n$-dimensional unit box with respect to the $n$-fold tensor product wavelet basis is also sparse and boundedly invertible. This greatly simplifies the implementation and improves the quantitative properties of an adaptive wavelet scheme to solve the multi-dimensional Poisson equation. The results extend to any second order partial differential operator with constant coefficients that defines a boundedly invertible operator.
\end{abstract}

Keywords Sparse representations - Tensor product approximation ·

Adaptive wavelet scheme $\cdot$ Riesz bases $\cdot$ Cubic Hermite splines

Mathematics Subject Classification (2000) $\quad 15 \mathrm{~A} 12 \cdot 15 \mathrm{~A} 69 \cdot 41 \mathrm{~A} 15 \cdot 41 \mathrm{~A} 25 \cdot$ $65 \mathrm{~N} 99 \cdot 65 \mathrm{~F} 50 \cdot 65 \mathrm{~T} 60$

\section{Introduction}

Let us denote $\mathrm{I}:=(0,1)$ and $\square:=\mathrm{I}^{n}$. In [9], we developed an adaptive tensor product wavelet method that for given $f \in H^{-1}(\square)$ solves the problem of finding $u \in H_{0}^{1}(\square)$

\footnotetext{
T. J. Dijkema

Department of Mathematics, Utrecht University, P.O. Box 80010, 3508 TA Utrecht, The Netherlands e-mail: t.j.dijkema@gmail.com

R. Stevenson $(\varangle)$

Korteweg-de Vries Institute for Mathematics, University of Amsterdam, Plantage Muidergracht 24, 1018 TV Amsterdam, The Netherlands

e-mail: r.p.stevenson@uva.nl
} 
such that

$$
a(u, v):=\int_{\square} c_{0} u v+\sum_{m=1}^{n} c_{m} \partial_{m} u \partial_{m} v=f(v) \quad\left(v \in H_{0}^{1}(\square)\right),
$$

where $c_{0} \geq 0$ and $c_{m}>0(m=1, \ldots, n)$ are constants. Actually, there we allowed homogeneous Dirichlet boundary conditions on only part of the boundary, but, as we will see, in this paper we need them on the whole of the boundary. General, possibly non-symmetric second order partial differential operators with constant coefficients will be considered at the end of Sect. 3 .

Using that

$$
H_{0}^{1}(\square)=H_{0}^{1}(\mathrm{I}) \otimes L_{2}(\mathrm{I}) \otimes \cdots \otimes L_{2}(\mathrm{I}) \cap \cdots \cap L_{2}(\mathrm{I}) \otimes \cdots \otimes L_{2}(\mathrm{I}) \otimes H_{0}^{1}(\mathrm{I}),
$$

we constructed a Riesz basis for $H_{0}^{1}(\square)$ by tensorizing univariate Riesz bases of wavelet type. Indeed, if $\Psi=\left\{\psi_{\lambda}: \lambda \in \nabla\right\}$ is a Riesz basis for $L_{2}$ (I) that, when normalized in $H^{1}(\mathrm{I})$, is a Riesz basis for $H_{0}^{1}(\mathrm{I})$, then, when normalized in $H^{1}(\square), \Psi \otimes \cdots \otimes \Psi$ is a Riesz basis for $H_{0}^{1}(\square)$. This holds true with Riesz constants that are bounded uniformly in $c_{0} \geq 0$ and $c_{m}>0(m=1, \ldots, n)$, when we equip $H_{0}^{1}(\square)$ with the energy norm $\|\cdot\|=a(\cdot, \cdot)^{\frac{1}{2}}$. These Riesz constants are even bounded uniformly in the space dimension $n$ if (and only if) $\Psi$ is an orthonormal basis for $L_{2}(\mathrm{I})$.

Denoting the resulting Riesz basis for $H_{0}^{1}(\square)$ as

$$
\boldsymbol{\Psi}:=\left\{\boldsymbol{\psi}_{\lambda}:=\otimes_{m=1}^{n} \psi_{\lambda_{m}} /\left\|\otimes_{m=1}^{n} \psi_{\lambda_{m}}\right\|: \lambda \in \nabla:=\nabla^{n}\right\}
$$

by writing $u=\mathbf{u}^{\top} \boldsymbol{\Psi}:=\sum_{\lambda \in \nabla} \mathbf{u}_{\lambda} \psi_{\lambda}$, and with $\mathbf{f}:=\left[f\left(\psi_{\lambda}\right)\right]_{\lambda \in \nabla}$, an equivalent formulation of (1) is

$$
\mathbf{A u}=\mathbf{f}
$$

The stiffness matrix A with respect to $\boldsymbol{\Psi}$ reads as

$\mathbf{A}=\mathbf{D}^{-1}\left(c_{0} \vec{M} \otimes \cdots \otimes \vec{M}+c_{1} \vec{A} \otimes \vec{M} \otimes \cdots \otimes \vec{M}+\cdots+c_{n} \vec{M} \otimes \cdots \otimes \vec{M} \otimes \vec{A}\right) \mathbf{D}^{-1}$, where $\mathbf{D}:=\operatorname{diag}\left[\left\|\otimes_{m=1}^{n} \psi_{\lambda_{m}}\right\|: \lambda \in \nabla\right]$, and

$$
\vec{A}:=\left[\int_{\mathrm{I}} \dot{\psi}_{\mu} \dot{\psi}_{\lambda}\right]_{\lambda, \mu \in \nabla} \text { and } \vec{M}:=\left[\int_{\mathrm{I}} \psi_{\mu} \psi_{\lambda}\right]_{\lambda, \mu \in \nabla}
$$

are the one-dimensional (unnormalized) stiffness and mass matrices, respectively. Here, and on other places, a (double) "dot" on top of a univariate function denotes its (second) derivative. A (double) "dot" on top of a linear space of univariate functions will denote the linear space of (second) derivatives of these functions. The aforementioned results about $\Psi$ being a Riesz basis for $H_{0}^{1}(\square)$ equipped with $\|\cdot\| \mid$ are equivalent 
to the matrix A defining a boundedly invertible mapping on $\ell_{2}(\nabla)$, with a condition number that is bounded uniformly in $c_{0} \geq 0$ and $c_{m}>0(m=1, \ldots, n)$ (and in $n$ if and only if $\Psi$ is $L_{2}(\mathrm{I})$-orthonormal). Another equivalent property is that for $\mathbf{v} \in \ell_{2}(\nabla)$ being an approximation to $\mathbf{u}$, it holds that

$$
\left\|u-\mathbf{v}^{\top} \boldsymbol{\Psi}\right\| \approx\|\mathbf{u}-\mathbf{v}\|_{\ell_{2}(\nabla)} .
$$

Here and in the remainder, with $C \lesssim D$ we will mean that $C$ can be bounded by a multiple of $D$, independently of parameters on which $C$ and $D$ may depend, possibly with the exception of the space dimension $n$. Obviously, $C \gtrsim D$ is defined as $D \lesssim C$, and $C \approx D$ as $C \lesssim D$ and $C \gtrsim D$.

In [9], we solved (2) with an adaptive wavelet Galerkin method introduced in [3] and later modified in [10]. Given a finite set $\boldsymbol{\Lambda} \subset \nabla$, let $\mathbf{I}_{\boldsymbol{\Lambda}}: \ell_{2}(\boldsymbol{\Lambda}) \rightarrow \ell_{2}(\boldsymbol{\nabla})$ denote the trivial embedding, so that its adjoint $\mathbf{P}_{\Lambda}: \ell_{2}(\nabla) \rightarrow \ell_{2}(\boldsymbol{\Lambda})$ is the restriction of a vector to its indices in $\Lambda$. With $\mathbf{A}_{\boldsymbol{\Lambda}}:=\mathbf{P}_{\boldsymbol{\Lambda}} \mathbf{A I}_{\boldsymbol{\Lambda}}$ and $\mathbf{f}_{\boldsymbol{\Lambda}}:=\mathbf{P}_{\boldsymbol{\Lambda}} \mathbf{f}$, the solution of $\mathbf{A}_{\boldsymbol{\Lambda}} \mathbf{u}_{\boldsymbol{\Lambda}}=\mathbf{f}_{\boldsymbol{\Lambda}}$ is known as the Galerkin approximation to $\mathbf{u}$ from $\ell_{2}(\boldsymbol{\Lambda})$. The idealized adaptive wavelet Galerkin scheme reads as follows:

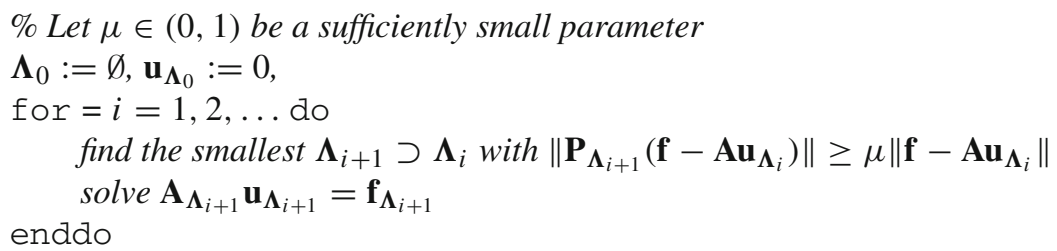

Note that the residual $\mathbf{f}-\mathbf{A} \mathbf{u}_{\boldsymbol{\Lambda}_{i}}$ plays the role of an a posteriori error estimator to guide a proper expansion of the set $\boldsymbol{\Lambda}_{i}$.

The above scheme cannot be performed exactly. First of all, generally $\mathbf{f}$ will be infinitely supported and thus has to be approximated. Secondly, with the available univariate wavelet bases, either $\vec{M}$ or $\vec{A}$ or both are not sparse, and so generally any column of $\mathbf{A}$ has infinitely many non-zeros. Thanks to the properties of wavelets, however, as being smooth and having vanishing moments, the sizes of the entries of $\vec{M}$ and $\vec{A}$, and thus of $\mathbf{A}$ do decay rapidly away from the diagonal. This property has been used to design an adaptive approximate matrix-vector multiplication routine APPLY in which the accuracy with which any column is approximated increases with the modulus of the corresponding entry in the vector. This APPLY routine is used both for approximate computation of the residual $\mathbf{f}-\mathbf{A} \mathbf{u}_{\boldsymbol{\Lambda}_{i}}$ and for the approximate multiplication with $\mathbf{A}_{\boldsymbol{\Lambda}_{i+1}}$ for the iterative solution of the Galerkin problem $\mathbf{A}_{\boldsymbol{\Lambda}_{i+1}} \mathbf{u}_{\boldsymbol{\Lambda}_{i+1}}=\mathbf{f}_{\boldsymbol{\Lambda}_{i+1}}$. Concerning the latter, note that generally the number of non-zero entries in $\mathbf{A}_{\boldsymbol{\Lambda}_{i+1}}$ is not of the order of $\# \boldsymbol{\Lambda}_{i+1}$.

The resulting practical scheme was shown to converge with the best possible rate in linear complexity. Moreover, since tensor product wavelets are applied, this rate is independent of the space dimension [8]. If (and only if) $\Psi$ is $L_{2}$ (I)-orthonormal, even the constant factor in the error bound that the adaptive scheme may lose compared to the corresponding best $N$-term approximations is independent of $n$. In future work, we will generalize the approach to non-product domains using domain decomposition techniques. 
Although the scheme has optimal computational complexity, quantitatively the application of the APPLY routine is very demanding, where this routine is also not easy to implement. This is the motivation to develop in this paper a univariate wavelet basis $\Psi$ such that both $\vec{A}$ and $\vec{M}$, and thus $\mathbf{A}$ are sparse. In this case, $\mathbf{A}$ can be applied exactly to a (finitely supported) vector at a cost that is linear in its support length. Since $\Psi$ will be a Riesz basis for $L_{2}(\mathrm{I})$ and, when normalized in $H^{1}(\mathrm{I})$, a Riesz basis for $H_{0}^{1}(\mathrm{I})$, the bi-infinite matrix $\mathbf{A}$, i.e., the representation of the operator defined in (1) with respect to the normalized tensor product basis, will be a boundedly invertible mapping, uniformly in $c_{0} \geq 0$ and $c_{m}>0(m=1, \ldots, n)$. Since $\Psi$, however, will not be $L_{2}$ (I)-orthonormal, the condition number of $\mathbf{A}$ will grow with the space dimension $n$.

Remark 1 We emphasize that with above wavelets, for any subset $\boldsymbol{\Lambda} \subset \boldsymbol{\nabla},\left.\mathbf{A}\right|_{\boldsymbol{\Lambda} \times \boldsymbol{\Lambda}}$ is sparse and well-conditioned, with a condition number bounded by that of $\mathbf{A}$. As a consequence, these wavelets may also find their application in non-adaptive sparse grid algorithms (e.g. see [1]). Indeed, with the usually applied hierarchical basis, neither $\left.\mathbf{A}\right|_{\boldsymbol{\Lambda} \times \boldsymbol{\Lambda}}$ is sparse, nor its condition number is bounded uniformly in $\boldsymbol{\Lambda}$.

Remark 2 When having univariate wavelets that lead to sparse $\vec{A}$ and $\vec{M}$, the stiffness matrix $\mathbf{A}$ corresponding to (1) is sparse because the coefficients $c_{i}$ are constants. For smooth, non-constant coefficients, the additional non-zeros outside the sparsity pattern of a constant coefficient operator will be much smaller, depending on the levels of the wavelets involved. For the residual computation inside the adaptive wavelet scheme, which is the quantitatively most demanding part, it can be envisaged that they can be ignored, possibly apart from those corresponding to some coarsest levels.

Remark 3 Instead of being satisfied with a stiffness matrix $\mathbf{A}$ that is sparse, one may think of searching a wavelet basis of $H_{0}^{1}(\square)$ such that the stiffness matrix is diagonal. This would mean that if $\mathbf{f}$ has a finite support $\boldsymbol{\Lambda} \subset \nabla$, then the exact solution of (1) is in the span of the wavelets with indices in $\boldsymbol{\Lambda}$. This seems hard, or perhaps impossible to realize on a bounded domain and for dimensions $n \geq 2$. We refer to [4] for a discussion of related issues on the domain $\mathbb{R}^{2}$.

Of course, in order to end up with a diagonal stiffness matrix, one can tensorize the univariate basis $\left\{\sqrt{2} \sin (k \pi x): k \in \mathbb{N}_{0}\right\}$. As shown in [8], with this approach, however, even for smooth $f$ generally only low convergence rates are possible.

\section{A first attempt: continuous piecewise smooth wavelets?}

We will search a collection of univariate wavelets $\Psi=\left\{\psi_{\lambda}: \lambda \in \nabla\right\}$ such that, with $|\lambda| \in \mathbb{N}_{0}$ denoting the level of $\psi_{\lambda}$ or that of $\lambda$,

(a) diam supp $\psi_{\lambda} \lesssim 2^{-|\lambda|}$,

(b) $\sup _{j, k \in \mathbb{N}_{0}} \#\left\{|\lambda|=j:\left[k 2^{-j},(k+1) 2^{-j}\right] \cap \operatorname{supp} \psi_{\lambda} \neq \emptyset\right\}<\infty$,

(c) $\Psi$ is Riesz basis for $L_{2}(\mathrm{I})$,

(d) $\left\{\psi_{\lambda} /\left\|\dot{\psi}_{\lambda}\right\|_{L_{2}(\mathrm{I})}: \lambda \in \nabla\right\}$ is a Riesz basis for $H_{0}^{1}(\mathrm{I})$,

(e) $\int_{\mathrm{I}} \dot{\psi}_{\lambda} \dot{\psi}_{\mu}=0$ when ||$\lambda|-| \mu||>M$,

(f) $\int_{\mathrm{I}} \psi_{\lambda} \psi_{\mu}=0$ when ||$\lambda|-| \mu||>M$, 
where $M \in N_{0}$ is some constant, that later will be chosen to be 1 . As a consequence, with respect to a level-wise partition of the wavelets, $\vec{A}$ and $\vec{M}$ will be block tridiagonal with, because of (a) and (b), sparse non-zero blocks. Note that under the assumptions (a) and (b), $\vec{A}$ and $\vec{M}$ are sparse if and only if (e) and (f), respectively, are valid. We will refer to the properties (a) and (b) by saying that the wavelets are (uniformly) local and that the collection of wavelets on each level is (uniformly) locally finite, respectively.

Proposition 1 If, in addition to (a)-(d), each wavelet is piecewise smooth with bounded piecewise first and second derivatives, then (e) requires that they are globally in $C^{1}$.

Proof Suppose the statement is wrong. For some $\mu \in \nabla$, let $\dot{\psi}_{\mu}$ have a jump in some $y \in \mathrm{I}$. Then there exists a $K=K(\mu) \geq M+|\mu|$ such that for all $\lambda \in \nabla$ with $|\lambda|>K$ and $\psi_{\lambda}(y) \neq 0$, it holds that supp $\psi_{\lambda} \subset \mathrm{I}$ and $\psi_{\mu}$ is smooth on supp $\psi_{\lambda} \backslash\{y\}$, where we used that $\psi_{\mu}$ is piecewise smooth. Then by (e), for those $\lambda$ we have

$$
0=\int_{\mathrm{I}} \dot{\psi}_{\lambda} \dot{\psi}_{\mu}=\left(\dot{\psi}_{\mu}\left(y^{-}\right)-\dot{\psi}_{\mu}\left(y^{+}\right)\right) \psi_{\lambda}(y)-\int_{\mathrm{I}} \ddot{\psi}_{\mu} \psi_{\lambda},
$$

and so ( $\mu$ is fixed),

$$
\left|\psi_{\lambda}(y)\right| \lesssim \int_{\mathrm{I}}\left|\psi_{\lambda}\right| \lesssim \sqrt{2^{-|\lambda|}}
$$

Writing $u \in L_{2}(\mathrm{I})$ as $u=\sum_{\lambda \in \nabla} c_{\lambda} \psi_{\lambda}$, (c) shows that $\|u\|_{L_{2}(\mathrm{I})}^{2} \approx \sum_{\lambda \in \nabla}\left|c_{\lambda}\right|^{2}$. When $u \in H_{0}^{1}(\mathrm{I})$, then (d) shows that this expansion converges also in $H^{1}(\mathrm{I})$, and thus in $L_{\infty}(\mathrm{I})$, i.e., that

$$
u(y)=\sum_{\left\{\lambda \in \nabla: \psi_{\lambda}(y) \neq 0\right\}} c_{\lambda} \psi_{\lambda}(y)
$$

Now by using (3) for $|\lambda|>K$, and the fact that $\left|\psi_{\lambda}(y)\right|<\infty$ for each of the finitely many other $\lambda \in \nabla$, an application of the Cauchy-Schwarz inequality shows that $|u(y)| \lesssim\|u\|_{L_{2}(\mathrm{I})}$, which inequality, however, is not valid on $H_{0}^{1}(\mathrm{I})$. We conclude that the wavelets have to be in $C^{1}$.

Remark 4 Proposition 1 confirms the well-known fact that the hierarchical basis is not a Riesz basis for $L_{2}(\mathrm{I})$. Indeed, this basis of continuous piecewise linears satisfies (a), (b), (d) and (e), where $\vec{A}$ is even diagonal, and thus it cannot satisfy (c).

Remark 5 Assuming (a), (b), (c), (e), and that each wavelet is piecewise smooth with bounded piecewise first and second derivatives, the above proof also shows that $\left\{\psi_{\lambda} /\left\|\psi_{\lambda}\right\|_{H^{1}(\mathrm{I})}: \lambda \in \nabla\right\}$ can be a Riesz basis for $H^{1}(\mathrm{I})$ (instead of $H_{0}^{1}(\mathrm{I})$ ) only if $\dot{\psi}_{\mu}(0)=\dot{\psi}_{\mu}(1)=0$ for all $\mu \in \nabla$. Indeed, suppose $\dot{\psi}_{\mu}$ does not vanish at the boundary, say at 0 . Then there exists a $K \geq M+|\mu|$ such that for all $\lambda \in \nabla$ with $|\lambda|>K$ 
and $\psi_{\lambda}(0) \neq 0$, it holds that supp $\psi_{\lambda} \subset[0,1)$ and $\psi_{\mu}$ is smooth on supp $\psi_{\lambda}$. Then by (e), for those $\lambda$ we have

$$
0=\int_{\mathrm{I}} \dot{\psi}_{\lambda} \dot{\psi}_{\mu}=-\dot{\psi}_{\mu}(0) \psi_{\lambda}(0)-\int_{\mathrm{I}} \ddot{\psi}_{\mu} \psi_{\lambda}
$$

and the same arguments as in the proof of Proposition 1 lead to a contradiction.

In view of having a rapidly converging wavelet expansion, for a wavelet basis for $H^{1}(\mathrm{I})$ the conditions $\dot{\psi}_{\mu}(0)=\dot{\psi}_{\mu}(1)=0$ are not desirable. In view of this, we restrict ourselves to the task of constructing a collection $\Psi$ such that (a)-(e) are valid, i.e., in particular such that $\left\{\psi_{\lambda} /\left\|\dot{\psi}_{\lambda}\right\|_{L_{2}(\mathrm{I})}: \lambda \in \nabla\right\}$ is a Riesz basis for $H_{0}^{1}(\mathrm{I})$.

\section{Biorthogonal multi-resolution analyses and wavelets}

In order to construct wavelets that, properly scaled, generate Riesz bases for a range of Sobolev spaces, in particular for $L_{2}$ (I) and $H_{0}^{1}$ (I) (cf. (c) and (d)), we will use the following well-known theorem (cf. [2,5,7]).

Theorem 1 (Biorthogonal space decompositions) Let

$$
V_{0} \subset V_{1} \subset \cdots \subset L_{2}(\mathrm{I}), \quad \tilde{V}_{0} \subset \tilde{V}_{1} \subset \cdots \subset L_{2}(\mathrm{I})
$$

be sequences of primal and dual spaces such that

$$
\operatorname{dim} V_{j}=\operatorname{dim} \tilde{V}_{j}<\infty \text { and } \alpha_{j}:=\inf _{0 \neq \tilde{v}_{j} \in \tilde{V}_{j}} \sup _{0 \neq v_{j} \in V_{j}} \frac{\left|\left\langle\tilde{v}_{j}, v_{j}\right\rangle_{L_{2}(\mathrm{I})}\right|}{\left\|\tilde{v}_{j}\right\|_{L_{2}(\mathrm{I})}\left\|v_{j}\right\|_{L_{2}(\mathrm{I})}} \gtrsim 1
$$

In addition, for some $0<\gamma<d$, let

$$
\inf _{v_{j} \in V_{j}}\left\|v-v_{j}\right\|_{L_{2}(\mathrm{I})} \lesssim 2^{-j d}\|v\|_{\mathcal{H}^{d}(\mathrm{I})} \quad\left(v \in \mathcal{H}^{d}(\mathrm{I})\right) \quad(\text { Jackson estimate }),
$$

and

$$
\left\|v_{j}\right\|_{\mathcal{H}^{s}(\mathrm{I})} \lesssim 2^{j s}\left\|v_{j}\right\|_{L_{2}(\mathrm{I})} \quad\left(v_{j} \in V_{j}, s \in[0, \gamma)\right) \quad \text { (Bernstein estimate) }
$$

where, for $s \in[0, d], \mathcal{H}^{s}(\mathrm{I})=\left[L_{2}(\mathrm{I}), \mathcal{H}^{d}(\mathrm{I})\right]_{s / d}$, and let similar estimates be valid at the dual side with $\left(\left(V_{j}\right)_{j}, d, \gamma, \mathcal{H}^{s}(\mathrm{I})\right)$ reading as $\left(\left(\tilde{V}_{j}\right)_{j}, \tilde{d}, \tilde{\gamma}, \tilde{\mathcal{H}}^{s}(\mathrm{I})\right)$.

Then, with $\Phi_{0}=\left\{\phi_{0, k}: k \in I_{0}\right\}$ being a basis for $V_{0}$ (scaling functions) and $\Psi_{j}=\left\{\psi_{j, k}: k \in J_{j}\right\}(j \in \mathbb{N})$ being uniform $L_{2}(\mathrm{I})$-Riesz bases for $W_{j}:=V_{j} \cap \tilde{V}_{j-1}^{\perp_{L_{2}(\mathrm{I})}}$ (wavelets), for $s \in(-\tilde{\gamma}, \gamma)$ the collection

$$
\Phi_{0} \cup \cup_{j \in \mathbb{N}} 2^{-s j} \Psi_{j}
$$

is a Riesz basis for $\mathcal{H}^{s}(\mathrm{I})$, where $\mathcal{H}^{s}(\mathrm{I}):=\left(\tilde{\mathcal{H}}^{-s}(\mathrm{I})\right)^{\prime}$ for $s<0$. 
In view of the notations introduced earlier, we denote $(j, k)$ also as $\lambda$, where $|\lambda|=j$, $\phi_{0, k}$ as $\psi_{0, k}$ and $I_{0} \cup \cup_{j \in \mathbb{N}} J_{j}$ as $\nabla$.

Remark 6 When $\operatorname{dim} V_{j}=\operatorname{dim} \tilde{V}_{j}<\infty$, the condition $\alpha_{j} \gtrsim 1$ in (4) is equivalent to the property that for uniform $L_{2}$ (I)-Riesz bases $\Phi_{j}$ and $\tilde{\Phi}_{j}$ for $V_{j}$ and $\tilde{V}_{j}$, respectively, $\left\langle\Phi_{j}, \tilde{\Phi}_{j}\right\rangle_{L_{2}(\mathrm{I})}^{-1}$ exists with a uniformly bounded spectral norm, or, equivalently, that $V_{j}$ and $\tilde{V}_{j}$ can be equipped with biorthogonal uniform $L_{2}(\mathrm{I})$-Riesz bases. In cases where these biorthogonal bases can be chosen to be both uniformly local, then under some mild additional condition, both the (primal) wavelets and the corresponding dual wavelets can be selected to be uniformly local (cf. [6]). In the application of Theorem 1 that we study in this paper, only the primal scaling functions and wavelets will be uniformly local.

\section{Biorthogonal cubic Hermite wavelets}

We shall select $\left(V_{j}\right)_{j},\left(\tilde{V}_{j}\right)_{j}$ that satisfy the conditions of Theorem 1 for some $\gamma>1$, where $\mathcal{H}^{d}(\mathrm{I})=H_{0}^{1}(\mathrm{I}) \cap H^{d}(\mathrm{I})$. In addition, $\left(V_{j}\right)_{j},\left(\tilde{V}_{j}\right)_{j}$ will be selected such that $V_{j} \subset C^{1}(\mathrm{I}) \cap H_{0}^{1}(\mathrm{I})$ and

$$
V_{j}+\ddot{V}_{j} \subset \tilde{V}_{j+1}
$$

As a consequence, using that for $|\mu|>0, \psi_{\mu} \perp_{L_{2}(\mathrm{I})} \tilde{V}_{|\mu|-1}$, for $|\mu|>|\lambda|+1$ we have

$$
\int_{\mathrm{I}} \psi_{\lambda} \psi_{\mu}=0, \quad \int_{\mathrm{I}} \dot{\psi}_{\lambda} \dot{\psi}_{\mu}=-\int_{\mathrm{I}} \ddot{\psi}_{\lambda} \psi_{\mu}=0,
$$

i.e., (e) and (f) are valid with $M=1$.

We will take $V_{j}$ to be the space of cubic Hermite splines satisfying first order homogeneous Dirichlet boundary conditions with respect to the $j+1$ times dyadically refined interval $\mathrm{I}=(0,1)$, and $\tilde{V}_{j}$ to be the space of piecewise cubics with respect to the $j$ times dyadically refined I, i.e.,

$$
\begin{aligned}
& V_{j}:=\prod_{k=0}^{2^{j+1}-1} P_{3}\left(k 2^{-(j+1)},(k+1) 2^{-(j+1)}\right) \cap C^{1}(\mathrm{I}) \cap H_{0}^{1}(\mathrm{I}), \\
& \tilde{V}_{j}:=\prod_{k=0}^{2^{j}-1} P_{3}\left(k 2^{-j},(k+1) 2^{-j}\right) .
\end{aligned}
$$

Clearly, with this choice (5) is satisfied (actually even $V_{j}+\ddot{V}_{j}=\tilde{V}_{j+1}$ ).

The dimension of $V_{j}$ is $4 \times 2^{j+1}-\left(2^{j+1}-1\right) 2-2=2^{j+2}=4 \times 2^{j}$, being the dimension of $\tilde{V}_{j}$. The second statement of the following theorem will be proved in Sect. 7. 
Theorem 2 It holds that $\operatorname{dim} V_{j}=\operatorname{dim} \tilde{V}_{j}$ and

$$
\inf _{j \in \mathbb{N}_{0}} \inf _{0 \neq \tilde{v}_{j} \in \tilde{V}_{j}} \sup _{0 \neq v_{j} \in V_{j}} \frac{\left|\left\langle\tilde{v}_{j}, v_{j}\right\rangle_{L_{2}(\mathrm{I})}\right|}{\left\|\tilde{v}_{j}\right\|_{L_{2}(\mathrm{I})}\left\|v_{j}\right\|_{L_{2}(\mathrm{I})}}>0 .
$$

In Sect. 5, we will construct uniform $L_{2}(\mathrm{I})$-Riesz bases $\Psi_{j}$ for $W_{j}=V_{j} \cap \tilde{V}_{j-1}^{\perp_{2}(\mathrm{I})}$. With $\Phi_{0}$ being some basis for $V_{0}$, an application of Theorems 1 and 2 yields the following result.

Corollary 1 Let $\mathcal{H}^{s}(\mathrm{I}):=\left[L_{2}(\mathrm{I}), H^{4}(\mathrm{I}) \cap H_{0}^{1}(\mathrm{I})\right]_{s / 4}$ for $s \in[0,4]$ and $\mathcal{H}^{s}(\mathrm{I}):=$ $\left(H^{-s}(\mathrm{I})\right)^{\prime}$ for $s<0$. Then for $s \in\left(-\frac{1}{2}, \frac{5}{2}\right)$, the collection $\Phi_{0} \cup \cup_{j \in \mathbb{N}} 2^{-s j} \Psi_{j}$ is a Riesz basis for $\mathcal{H}^{s}(\mathrm{I})$.

Remark 7 It is known (e.g. see [11]) that for $s \in[1,4], \mathcal{H}^{s}(\mathrm{I})=H^{s}(\mathrm{I}) \cap H_{0}^{1}(\mathrm{I})$ and that for $s \in[0,1] \backslash\left\{\frac{1}{2}\right\}, \mathcal{H}^{s}(\mathrm{I})=H_{0}^{s}(\mathrm{I})$, the latter space being equal to $H^{s}$ (I) for $s \in\left[0, \frac{1}{2}\right)$.

The wavelets that we are going to construct in Sect. 5 will be uniformly local and will be such that the collections $\Psi_{j}$ that span the spaces $W_{j}=V_{j} \cap \tilde{V}_{j-1}^{\perp_{L_{2}(I)}}$ are uniformly locally finite, i.e., the conditions (a) and (b) formulated in the previous section are valid. Since by construction (e) and (f) hold, and (c) and (d) are special cases of Corollary 1, we conclude that all conditions (a)-(f) formulated in Sect. 2 are valid.

Note that due to the absence of boundary conditions incorporated in the definition of $\tilde{V}_{j}$, all wavelets, i.e., any element of $\Psi_{j}$, has 4 vanishing moments. This is very convenient for constructing sparse approximations to $\mathbf{f}=\left[f\left(\psi_{\lambda}\right)\right]_{\lambda \in \nabla}$.

Remark 8 In addition to (e) and (f) we have

$$
\int_{\mathrm{I}} \psi_{\lambda} \dot{\psi}_{\mu}=0 \text { when }|| \lambda|-| \mu||>1 \text {. }
$$

For $|\lambda|-|\mu|>1$, this follows from the fact that $\dot{V}_{j} \subset \tilde{V}_{j+1}$, and for $|\mu|-|\lambda|>1$ by additionally using integration by parts and the first order homogeneous Dirichlet boundary conditions. A consequence is that for any constants $\left(a_{\alpha, \beta}\right)_{|\alpha|,|\beta| \leq 1}$, the representation, with respect to the properly scaled wavelet basis $\Psi$, of the problem of finding $u \in H_{0}^{1}(\square)$ such that for given $f \in H^{-1}(\square)$,

$$
\int_{\square} \sum_{|\alpha|,|\beta| \leq 1} a_{\alpha, \beta} \partial^{\alpha} u \partial^{\beta} v=f(v) \quad\left(v \in H_{0}^{1}(\square)\right),
$$

is of the form

$$
\mathbf{A u}=\mathbf{f}
$$


where $\mathbf{A}$ is sparse. Indeed, also first order partial derivatives or mixed second order partial derivatives lead to a tensor product of sparse matrices. The matrix $\mathbf{A}$ is boundedly invertible whenever the constants $\left(a_{\alpha, \beta}\right)_{|\alpha|,|\beta| \leq 1}$ are such that (9) defines a boundedly invertible operator between $H_{0}^{1}(\square)$ and $H^{-1}(\square)$. For cases where $\mathbf{A}$ is not symmetric positive definite, a possibility to solve (10) is to apply the adaptive wavelet Galerkin scheme to the normal equations.

Remark 9 Besides the cubic Hermite splines, we also tried the following maximally smooth spline options for the sequence $\left(V_{j}\right)_{j}$ :

$$
\begin{aligned}
& \text { (i). } V_{j}:=\prod_{k=0}^{2^{j+1}-1} P_{2}\left(k 2^{-(j+1)},(k+1) 2^{-(j+1)}\right) \cap C^{1}(\mathrm{I}) \cap H_{0}^{1}(\mathrm{I}), \\
& \text { (ii). } V_{j}:=\prod_{k=0}^{2^{j+1}-1} P_{3}\left(k 2^{-(j+1)},(k+1) 2^{-(j+1)}\right) \cap C^{2}(\mathrm{I}) \cap H_{0}^{1}(\mathrm{I}), \\
& \text { (iii). } V_{j}:=\prod_{k=0}^{2^{j+1}-1} P_{4}\left(k 2^{-(j+1)},(k+1) 2^{-(j+1)}\right) \cap C^{3}(\mathrm{I}) \cap H_{0}^{2}(\mathrm{I}) .
\end{aligned}
$$

In view of (5), in case (i) we have that $\ddot{V}_{j}=\prod_{k=0}^{2^{j+1}-1} P_{0}\left(k 2^{-(j+1)},(k+1) 2^{-(j+1)}\right)$ and $V_{j} \cap \ddot{V}_{j}=\{0\}$. When choosing $\tilde{V}_{j+1}=V_{j}+\ddot{V}_{j}$, it holds that $\operatorname{dim} V_{j}=2^{j+1}=$ $\operatorname{dim} \tilde{V}_{j}$, but as one may verify, $\alpha_{j}$ from (4) is zero for any $j \in \mathbb{N}_{0}$. Since $\alpha_{j} \gtrsim 1$ is a necessary condition for the wavelets to generate a Riesz basis for $L_{2}$ (I), with this choice (a)-(f) cannot be realized.

In case (ii), we have that $\ddot{V}_{j}=\prod_{k=0}^{2^{j+1}-1} P_{1}\left(k 2^{-(j+1)},(k+1) 2^{-(j+1)}\right) \cap C(\mathrm{I})$ and $V_{j} \cap \ddot{V}_{j}=\{0\}$. When choosing $\tilde{V}_{j+1}=V_{j}+\ddot{V}_{j}$, we have $\operatorname{dim} \tilde{V}_{j}=2\left(2^{j}+1\right)=$ $\operatorname{dim} V_{j}+1$, and Theorem 1 cannot be applied.

In case (iii), we have that $\ddot{V}_{j} \subsetneq Z_{j}:=\prod_{k=0}^{2^{j+1}-1} P_{2}\left(k 2^{-(j+1)},(k+1) 2^{-(j+1)}\right) \cap$ $C^{1}(\mathrm{I})$ and $V_{j} \cap \ddot{V}_{j} \subset V_{j} \cap Z_{j}=\{0\}$. Choosing $\tilde{V}_{j+1}=V_{j}+\ddot{V}_{j}$, we have $\operatorname{dim} V_{j}=$ $2^{j+1}=\operatorname{dim} \tilde{V}_{j}$, but, as we verified numerically, $\alpha_{j} \downarrow 0$ for $j \rightarrow 0$.

\section{Construction of the wavelets}

With $V_{j}$ and $\tilde{V}_{j}$ from (6) and (7), we construct uniform $L_{2}(\mathrm{I})$-Riesz bases $\Psi_{j+1}$ for $V_{j+1} \cap \tilde{V}_{j}^{\perp_{L_{2}(\mathrm{I})}}$, which are also uniformly local and uniformly locally finite.

Let $\phi^{(1)}, \phi^{(2)} \in P_{3}(-1,0) \times P_{3}(0,1) \cap C^{1}(-1,1)$ be defined by

$$
\begin{aligned}
\phi^{(1)}( \pm 1) & =0, & \dot{\phi}^{(2)}( \pm 1) & =0, \\
\phi^{(1)}(0) & =1, & \dot{\phi}^{(2)}(0) & =1, \\
\dot{\phi}^{(1)}(0)=\dot{\phi}^{(1)}( \pm 1) & =0, & \phi^{(2)}(0)=\phi^{(2)}( \pm 1) & =0 .
\end{aligned}
$$




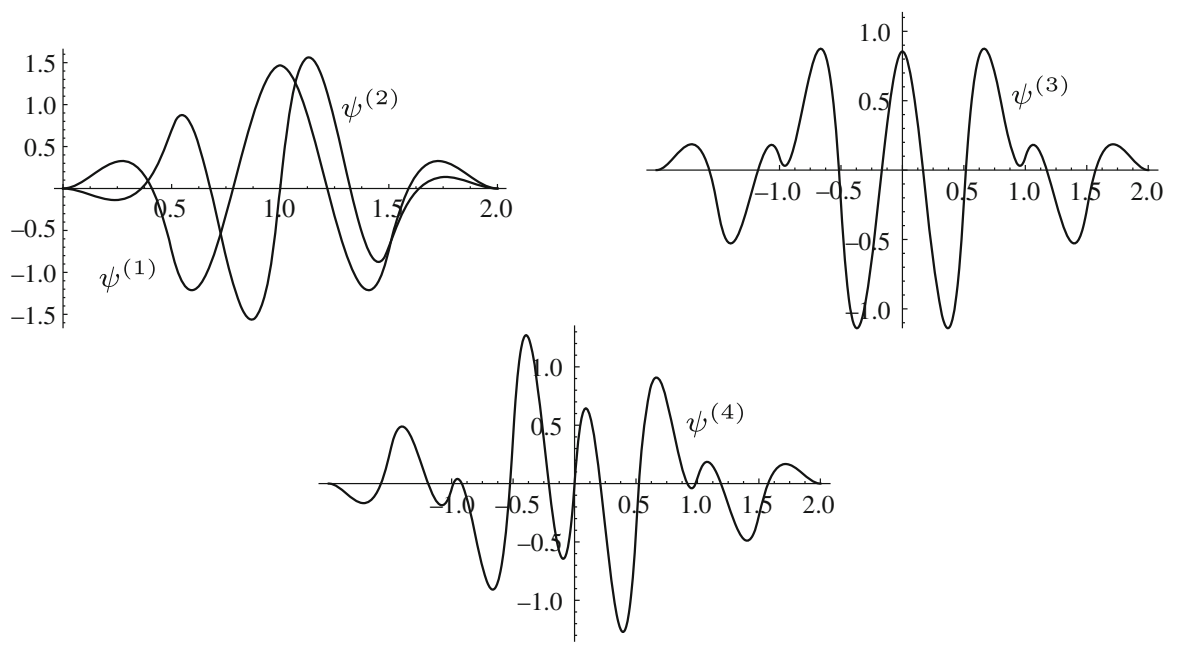

Fig. 1 The wavelets $\psi^{(1)}, \psi^{(2)}, \psi^{(3)}$ and $\psi^{(4)}$, normalized in $L_{2}$

Integer translates of $\phi^{(1)}, \phi^{(2)}$ span the space of $C^{1}$ piecewise cubics with respect to the pieces $[k, k+1](k \in \mathbb{Z})$. With

$$
\phi_{j, k}^{(i)}:=\sqrt{2^{j+1}} \phi^{(i)}\left(2^{j+1} \cdot-k\right)
$$

the collection

$$
\Phi_{j}:=\left\{\phi_{j, k}^{(1)}: k \in\left\{1,2, \ldots, 2^{j+1}-1\right\}\right\} \cup\left\{\left.\phi_{j, k}^{(2)}\right|_{\mathrm{I}}: k \in\left\{0,1, \ldots, 2^{j+1}\right\}\right\}
$$

is a uniform $L_{2}(\mathrm{I})$-Riesz basis for $V_{j}$ from (6).

We construct 4 types of "mother wavelets". These functions are $C^{1}$ piecewise cubics with respect to the pieces $\left[k, k+\frac{1}{2}\right]\left(k \in \frac{1}{2} \mathbb{Z}\right)$, i.e., they are in the span of $\left\{\phi^{(i)}(2 \cdot-k): i \in\{1,2\}, k \in \mathbb{Z}\right\}$, and they are $L_{2}(\mathbb{R})$-orthogonal to $\prod_{k \in 2 \mathbb{Z}} P_{3}(k, k+2)$ (Fig 1).

We seek the first two types of the form

$$
\begin{aligned}
\psi^{(1)} & :=\sum_{k=1}^{3} a_{k}^{(1)} \phi^{(1)}(2 \cdot-k)+\sum_{k=1}^{3} b_{k}^{(1)} \phi^{(2)}(2 \cdot-k), \\
\psi^{(2)} & :=\sum_{k=1}^{3} a_{k}^{(2)} \phi^{(1)}(2 \cdot-k)+\sum_{k=1}^{3} b_{k}^{(2)} \phi^{(2)}(2 \cdot-k),
\end{aligned}
$$

meaning that their support is $[0,2]$. Up to a scaling, these functions are uniquely determined by imposing that they are orthogonal to $P_{3}(0,2)$ and that $\psi^{(1)}(\cdot-1)$ is even and $\psi^{(2)}(\cdot-1)$ is odd. The coefficients $a_{k}^{(i)}, b_{k}^{(i)}(i \in\{1,2\})$ can be found in Table 1. 
Table 1 Coefficients for the construction of wavelets

\begin{tabular}{cccccccc}
\hline$k$ & -3 & -2 & -1 & 0 & 1 & 2 & 3 \\
\hline$a_{k}^{(1)}$ & - & - & - & - & $-\frac{2}{15}$ & $\frac{4}{15}$ & $-\frac{2}{15}$ \\
$b_{k}^{(1)}$ & - & - & - & - & -1 & 0 & 1 \\
$a_{k}^{(2)}$ & - & - & - & - & $\frac{7}{39}$ & 0 & $-\frac{7}{39}$ \\
$b_{k}^{(2)}$ & - & - & - & - & 1 & $\frac{44}{13}$ & 1 \\
$a_{k}^{(3)}$ & $-\frac{4595}{13728}$ & $\frac{7}{65}$ & $-\frac{18737}{68640}$ & 1 & $-\frac{18737}{68640}$ & $\frac{7}{65}$ & $-\frac{4595}{13728}$ \\
$b_{k}^{(3)}$ & $-\frac{68741}{22880}$ & $-\frac{69}{40}$ & $-\frac{204701}{22880}$ & 0 & $\frac{204701}{22880}$ & $\frac{69}{40}$ & $\frac{68741}{22880}$ \\
$a_{k}^{(4)}$ & $\frac{417}{22880}$ & $-\frac{7}{2340}$ & $\frac{5443}{205920}$ & 0 & $-\frac{5443}{205920}$ & $\frac{7}{2340}$ & $-\frac{417}{22880}$ \\
$b_{k}^{(4)}$ & $\frac{723}{4576}$ & $\frac{1}{8}$ & $\frac{8153}{13728}$ & $\frac{1}{2}$ & $\frac{8153}{13728}$ & $\frac{1}{8}$ & $\frac{723}{4576}$ \\
\hline
\end{tabular}

We seek the third and fourth type of the form

$$
\begin{aligned}
\psi^{(3)} & :=\sum_{k=-3}^{3} a_{k}^{(3)} \phi^{(1)}(2 \cdot-k)+\sum_{k=-3}^{3} b_{k}^{(3)} \phi^{(2)}(2 \cdot-k), \\
\psi^{(4)} & :=\sum_{k=-3}^{3} a_{k}^{(4)} \phi^{(1)}(2 \cdot-k)+\sum_{k=-3}^{3} b_{k}^{(4)} \phi^{(2)}(2 \cdot-k),
\end{aligned}
$$

meaning that their support is $[-2,2]$. Up to a scaling, these functions are uniquely determined by imposing that they are orthogonal to $P_{3}(-2,0) \times P_{3}(0,2)$, that $\psi^{(3)}$ is even and $\psi^{(4)}$ is odd and, in order to create a more sparse mass matrix, that they are orthogonal to $\psi^{(1)}(\cdot-k)$ and $\psi^{(2)}(\cdot-k)(k \in 2 \mathbb{Z})$. The coefficients $a_{k}^{(i)}, b_{k}^{(i)}$ $(i \in\{3,4\})$ can be found in Table 1 .

With

$$
\psi_{j+1, k}^{(i)}:=\sqrt{2^{j+1}} \psi^{(i)}\left(2^{j+1} \cdot-k\right),
$$

by construction, the collection

$$
\begin{aligned}
& \Psi_{j+1}:=\left\{\psi_{j+1, k}^{(i)}: i \in\{1,2\}, k \in\left\{0,2, \ldots, 2^{j+1}-2\right\}\right\} \\
& \cup\left\{\psi_{j+1, k}^{(3)}: k \in\left\{2,4, \ldots, 2^{j+1}-2\right\}\right\} \cup\left\{\left.\psi_{j+1, k}^{(4)}\right|_{\mathrm{I}}: k \in\left\{0,2, \ldots, 2^{j+1}\right\}\right\}
\end{aligned}
$$

is contained in $W_{j+1}=V_{j+1} \cap \tilde{V}_{j}^{\perp_{2}(\mathrm{I})}$, where its cardinality, being $2^{j+2}$, is equal to the dimension of this space, i.e., the collection spans $W_{j+1}$.

With $\bar{\psi}_{1}:=\psi_{1}, \bar{\psi}_{2}:=\psi_{2}, \bar{\psi}_{3}:=\left.\psi_{3}\right|_{[0,2]}, \bar{\psi}_{4}:=\left.\psi_{4}\right|_{[0,2]}, \bar{\psi}_{5}:=\left.\psi_{3}(\cdot-2)\right|_{[0,2]}$ and $\bar{\psi}_{6}:=\left.\psi_{4}(\cdot-2)\right|_{[0,2]}$, a numerical calculation reveals that the "element mass matrix" matrix 


$$
\left[\left\langle\bar{\psi}_{i}, \bar{\psi}_{j}\right\rangle_{L_{2}(0,2)}\right]_{1 \leq i, j \leq 6}=\left[\begin{array}{cccccc}
\frac{2467613}{3603600} & \frac{400733}{10810800} & 0 & 0 & -\frac{923411}{3603600} & \frac{137987}{10810800} \\
\frac{400733}{10810800} & \frac{7841}{3603600} & 0 & 0 & -\frac{137987}{10810800} & \frac{20431}{32432400} \\
0 & 0 & \frac{52}{1575} & 0 & 0 & 0 \\
0 & 0 & 0 & \frac{704}{10647} & 0 & 0 \\
-\frac{923411}{3603600} & -\frac{137987}{10810800} & 0 & 0 & \frac{2467613}{3603600} & -\frac{400733}{10810800} \\
\frac{137987}{10810800} & \frac{20431}{32432400} & 0 & 0 & -\frac{400733}{10810800} & \frac{7841}{3603600}
\end{array}\right]
$$

is positive definite. As a consequence, for any $J \subset 2 \mathbb{Z}$, and any subset

$$
\Sigma \subset\left\{\psi^{(i)}(\cdot-k): i \in\{1, \ldots, 4\}, k \in 2 \mathbb{Z}\right\}
$$

of functions that do not identically vanish on $G:=\cup_{k \in J}(k, k+2),\left\{\left.\sigma\right|_{G}: \sigma \in \Sigma\right\}$ is a $L_{2}(G)$-Riesz basis of its span with a condition number that can be bounded on an absolute multiple of the condition number of $\left[\left\langle\bar{\psi}_{i}, \bar{\psi}_{j}\right\rangle_{L_{2}(0,2)}\right]_{1 \leq i, j \leq 6}$. This follows from the observation that

$$
\left\langle\sum_{\sigma \in \Sigma} c_{\sigma} \sigma, \sum_{\tau \in \Sigma} c_{\tau} \tau\right\rangle_{L_{2}(G)}=\sum_{k \in J}\left\langle\left.\sum_{\sigma \in \Sigma} c_{\sigma} \sigma\right|_{(k, k+2)},\left.\sum_{\tau \in \Sigma} c_{\tau} \tau\right|_{(k, k+2)}\right\rangle_{L_{2}(k, k+2)} .
$$

Since the same holds true for the dilated functions, we conclude that (13) defines a uniform $L_{2}$ (I)-Riesz basis for $V_{j+1} \cap \tilde{V}_{j}^{\perp_{L_{2}(\mathrm{I})}}$.

\section{Condition numbers}

A result of Corollary 1 is that $\Phi_{0} \cup \cup_{j \in \mathbb{N}} \Psi_{j}$, where $\Phi_{0}$ and $\Psi_{j}$ are as in (12) and (13), respectively, forms, when normalized in $L_{2}$ (I) or $H^{1}(\mathrm{I})$, a Riesz basis for $L_{2}(\mathrm{I})$ and $H_{0}^{1}(\mathrm{I})$, respectively. In particular, this shows that the condition numbers of the mass matrix and the normalized stiffness matrix are bounded. In various estimates, the values of these condition numbers play a role. Since it is not feasible to compute the actual condition numbers of the infinite dimensional matrices, instead we computed those of

$$
\vec{A}_{J}:=\left[\frac{\int_{\mathrm{I}} \dot{\psi}_{\mu} \dot{\psi}_{\lambda}}{\left\|\dot{\psi}_{\mu}\right\|_{L_{2}(\mathrm{I})}\left\|\dot{\psi}_{\lambda}\right\|_{L_{2}(\mathrm{I})}}\right]_{\lambda, \mu \in \nabla,|\lambda|,|\mu| \leq J} \text { and } \vec{M}_{J}:=\left[\int_{\mathrm{I}} \psi_{\mu} \psi_{\lambda}\right]_{\lambda, \mu \in \nabla,|\lambda|,|\mu| \leq J} .
$$

The condition numbers of these matrices, which are bounded uniformly in $J$, are shown in Fig. 2.

Also, we computed the condition number of the mass matrix of wavelets on one level, i.e., the condition number of the matrix $\left[\int_{\mathrm{I}} \psi_{\mu} \psi_{\lambda}\right]_{\lambda, \mu \in \nabla,|\lambda|=|\mu|=J}$. Numerical results show that the value of this condition number is bounded by 2.2 uniformly in $J$. 

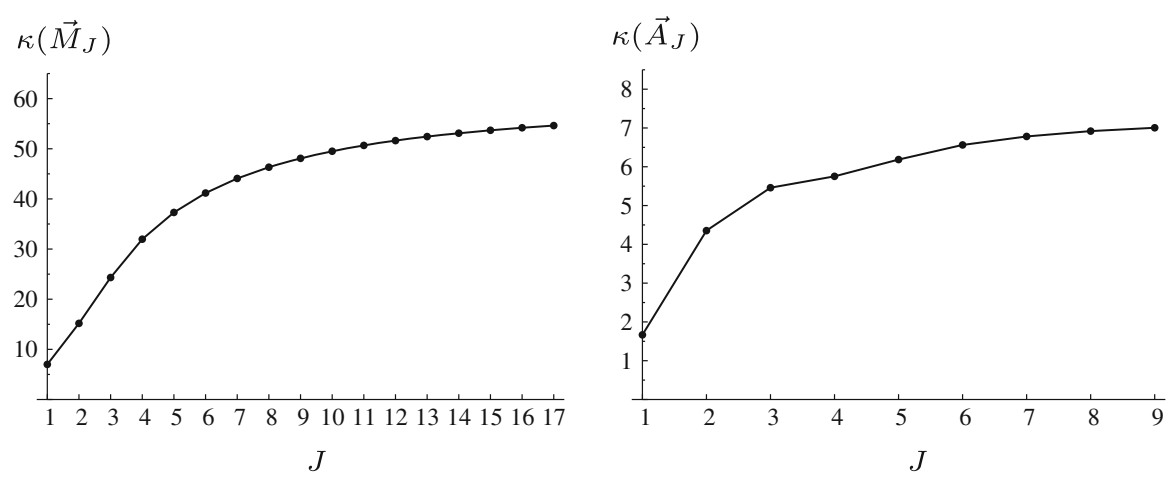

Fig. 2 Condition number of the mass matrix $\vec{M}_{J}$ (left) and the stiffness matrix $\vec{A}_{J}$ (right)

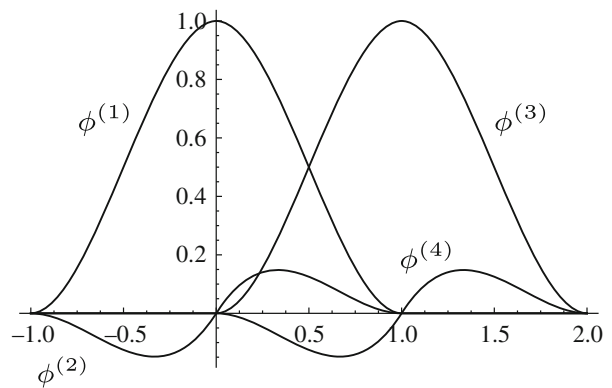

Fig. $3\left\{\phi^{(1)}, \phi^{(2)}, \phi^{(3)}, \phi^{(4)}\right\}$ from (14)

\section{Proof of Theorem 2}

In view of Remark 6, it suffices to construct uniform $L_{2}$ (I)-Riesz bases $\Phi_{j}$ and $\tilde{\Phi}_{j}$ for $V_{j}$ from (6) and $\tilde{V}_{j}$ from (7), respectively, such that $\left\langle\Phi_{j}, \tilde{\Phi}_{j}\right\rangle_{L_{2}(\mathrm{I})}$ is invertible, with an inverse that is bounded uniformly in $j$.

With $\phi^{(1)}$ and $\phi^{(2)}$ from (11), and $\phi^{(3)}:=\phi^{(1)}(\cdot-1)$ and $\phi^{(4)}:=\phi^{(2)}(\cdot-1)$,

$$
\left\{\phi^{(i)}(\cdot-k): i \in\{1, \ldots, 4\}, k \in 2 \mathbb{Z}\right\}
$$

spans the space of $C^{1}$ piecewise cubics with respect to the pieces $[k, k+1](k \in \mathbb{Z})$, see Fig. 3. With $\tilde{\phi}^{(i)}(x):=\left.(x-1)^{i-1}\right|_{[0,2]}$, obviously

$$
\left\{\tilde{\phi}^{(i)}(\cdot-k): i \in\{1, \ldots, 4\}, k \in 2 \mathbb{Z}\right\}
$$

spans $\prod_{k \in 2 \mathbb{Z}} P_{3}(k, k+2)$, see Fig. 4 .

We apply a number of basis transformation at primal and dual side. First we update $\phi^{(1)}, \phi^{(2)}$ with multiples of $\phi^{(3)}, \phi^{(3)}(\cdot+2), \phi^{(4)}, \phi^{(4)}(\cdot+2)$, and $\tilde{\phi}^{(1)}, \tilde{\phi}^{(2)}$ with multiples of $\tilde{\phi}^{(3)}$ and $\tilde{\phi}^{(4)}$ in such a way that the new $\phi^{(1)}, \phi^{(2)}$ are orthogonal to $\tilde{\phi}^{(3)}(\cdot-k)$ and $\tilde{\phi}^{(4)}(\cdot-k)(k \in 2 \mathbb{Z})$, and the new $\tilde{\phi}^{(1)}, \tilde{\phi}^{(2)}$ are orthogonal to $\phi^{(3)}$ 


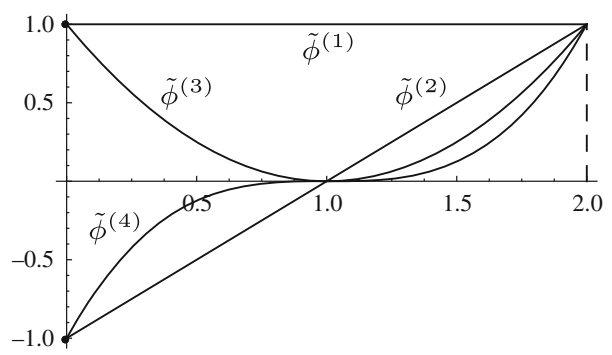

Fig. $4\left\{\tilde{\phi}^{(1)}, \tilde{\phi}^{(2)}, \tilde{\phi}^{(3)}, \tilde{\phi}^{(4)}\right\}$ from (15)

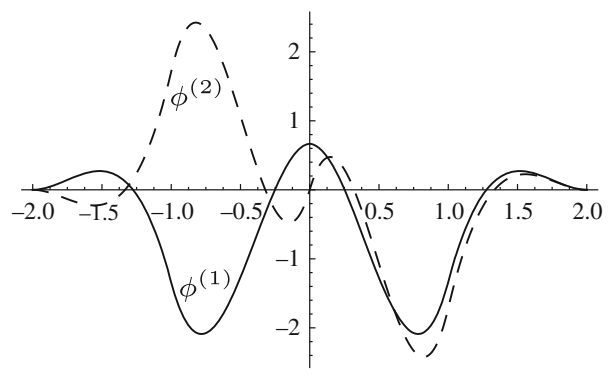

Fig. 5 New primal functions $\left\{\phi^{(1)}, \phi^{(2)}\right\}$

and $\phi^{(4)}$. In particular, we redefine

$$
\begin{aligned}
& {\left[\begin{array}{l}
\phi^{(1)} \\
\phi^{(2)}
\end{array}\right]:=\left[\begin{array}{l}
\phi^{(1)} \\
\phi^{(2)}
\end{array}\right]+\left[\begin{array}{rrrr}
-2 & -2 & -\frac{45}{4} & \frac{45}{4} \\
-\frac{1}{4} & \frac{1}{4} & \frac{5}{4} & \frac{5}{4}
\end{array}\right]\left[\begin{array}{c}
\phi^{(3)} \\
\phi^{(3)}(\cdot+2) \\
\phi^{(4)} \\
\phi^{(4)}(\cdot+2)
\end{array}\right],} \\
& {\left[\begin{array}{l}
\tilde{\phi}^{(1)} \\
\tilde{\phi}^{(2)}
\end{array}\right]:=\left[\begin{array}{l}
\tilde{\phi}^{(1)} \\
\tilde{\phi}^{(2)}
\end{array}\right]+\left[\begin{array}{rr}
-\frac{15}{2} & 0 \\
0 & -\frac{7}{2}
\end{array}\right]\left[\begin{array}{c}
\tilde{\phi}^{(3)} \\
\tilde{\phi}^{(4)}
\end{array}\right] .}
\end{aligned}
$$

To make two more inner products between local primal and dual functions zero, next we redefine

$$
\left[\begin{array}{l}
\tilde{\phi}^{(1)} \\
\tilde{\phi}^{(2)}
\end{array}\right]:=\left[\begin{array}{cc}
-\frac{1}{4} & \frac{15}{16} \\
-\frac{1}{4} & -\frac{15}{16}
\end{array}\right]\left[\begin{array}{l}
\tilde{\phi}^{(1)} \\
\tilde{\phi}^{(2)}
\end{array}\right] .
$$

Furthermore, we multiply $\phi^{(1)}$ with $\frac{2}{3}$ and $\phi^{(2)}$ with $\frac{48}{7}$. Finally, we redefine

$$
\left[\begin{array}{c}
\tilde{\phi}^{(1)} \\
\tilde{\phi}^{(2)}
\end{array}\right]:=\left[\begin{array}{rr}
1 & 1 \\
1 & -1
\end{array}\right]\left[\begin{array}{c}
\tilde{\phi}^{(1)} \\
\tilde{\phi}^{(2)}(\cdot+2)
\end{array}\right] .
$$

By the last transformation, as $\phi^{(1)}\left(\phi^{(2)}\right)$ the function $\tilde{\phi}^{(1)}\left(\tilde{\phi}^{(2)}\right)$ is even (odd).

The newly defined primal and dual scaling functions are illustrated in Figs. 5 and 6 , respectively. Note that our transformations did not change the spans of the collections (14) and (15). 


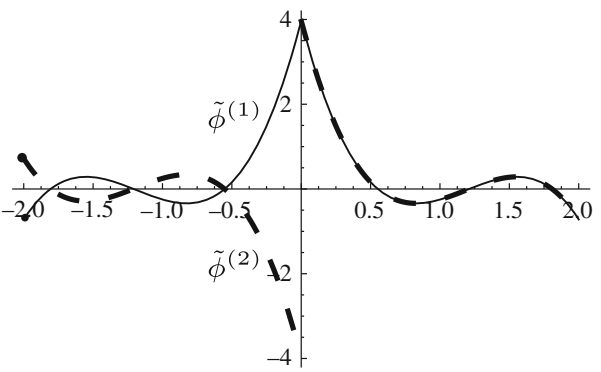

Fig. 6 New dual functions $\left\{\tilde{\phi}^{(1)}, \tilde{\phi}^{(2)}\right\}$

A direct computation shows that

$$
\left\langle\begin{array}{c}
\phi^{(1)} \\
\phi^{(2)} \\
\phi^{(1)}(\cdot-2) \\
\phi^{(2)}(\cdot-2) \\
\phi^{(3)} \\
\phi^{(4)}
\end{array}\right],\left[\begin{array}{c}
\tilde{\phi}^{(1)} \\
\tilde{\phi}^{(2)} \\
\tilde{\phi}^{(1)}(\cdot-2) \\
\tilde{\phi}^{(2)}(\cdot-2) \\
\tilde{\phi}^{(3)} \\
\tilde{\phi}^{(4)}
\end{array}\right]_{L_{2}(0,2)}=\left[\begin{array}{rrrr|rr}
\frac{1}{2} & \frac{1}{2} & 0 & 0 & 0 & 0 \\
\frac{1}{2} & \frac{1}{2} & \frac{1}{14} & -\frac{1}{14} & 0 & 0 \\
0 & 0 & \frac{1}{2} & -\frac{1}{2} & 0 & 0 \\
-\frac{1}{14} & -\frac{1}{14} & -\frac{1}{2} & \frac{1}{2} & 0 & 0 \\
\hline 0 & 0 & 0 & 0 & \frac{2}{15} & 0 \\
0 & 0 & 0 & 0 & 0 & \frac{2}{105}
\end{array}\right] .
$$

With

$$
\phi_{j, k}^{(i)}:=\sqrt{2^{j+1}} \phi^{(i)}\left(2^{j+1} \cdot-k\right), \quad \tilde{\phi}_{j, k}^{(i)}:=\sqrt{2^{j+1}} \tilde{\phi}^{(i)}\left(2^{j+1} \cdot-k\right)
$$

and

$$
\begin{aligned}
& \Phi_{j}^{(1)}:=\left\{\phi_{j, k}^{(1)}: k \in\left\{2, \ldots, 2^{j+1}-2\right\}\right\} \cup\left\{\left.\phi_{j, k}^{(2)}\right|_{\mathrm{I}}: k \in\left\{0,2, \ldots, 2^{j+1}\right\}\right\}, \\
& \Phi_{j}^{(2)}:=\left\{\phi_{j, k}^{(i)}: i \in\{3,4\}, k \in\left\{0,2, \ldots, 2^{j+1}-2\right\}\right\}, \\
& \tilde{\Phi}_{j}^{(1)}:=\left\{\tilde{\phi}_{j, k}^{(1)}: k \in\left\{2, \ldots, 2^{j+1}-2\right\}\right\} \cup\left\{\left.\tilde{\phi}_{j, k}^{(2)}\right|_{\mathrm{I}}: k \in\left\{0,2, \ldots, 2^{j+1}\right\}\right\}, \\
& \tilde{\Phi}_{j}^{(2)}:=\left\{\tilde{\phi}_{j, k}^{(i)}: i \in\{3,4\}, k \in\left\{0,2, \ldots, 2^{j+1}-2\right\}\right\},
\end{aligned}
$$

the collections $\Phi_{j}:=\Phi_{j}^{(1)} \cup \Phi_{j}^{(2)}$ and $\tilde{\Phi}_{j}:=\tilde{\Phi}_{j}^{(1)} \cup \tilde{\Phi}_{j}^{(2)}$ are uniform $L_{2}$ (I)-Riesz bases for $V_{j}$ and $\tilde{V}_{j}$, respectively. Indeed, one verifies that span $\Phi_{j} \subset V_{j}$, span $\tilde{\Phi}_{j} \subset \tilde{V}_{j}$ and that $\# \Phi_{j}=\operatorname{dim} V_{j}=2^{j+1}=\operatorname{dim} \tilde{V}_{j}=\# \tilde{\Phi}_{j}$. From the local supports and the proper normalization of the basis functions, one also easily verifies that for any coefficient vector $\mathbf{c}_{j}$ of the appropriate size, $\left\|\mathbf{c}_{j}^{\top} \Phi_{j}\right\|_{L_{2}(\mathrm{I})} \lesssim\left\|\mathbf{c}_{j}\right\|_{\ell_{2}}$ and $\left\|\mathbf{c}_{j}^{\top} \tilde{\Phi}_{j}\right\|_{L_{2}(\mathrm{I})} \lesssim\left\|\mathbf{c}_{j}\right\|_{\ell_{2}}$.

Instead of a direct verification that also $\left\|\mathbf{c}_{j}^{\top} \Phi_{j}\right\|_{L_{2}(\mathrm{I})} \gtrsim\left\|\mathbf{c}_{j}\right\|_{\ell_{2}}$ and $\left\|\mathbf{c}_{j}^{\top} \tilde{\Phi}_{j}\right\|_{L_{2}(\mathrm{I})} \gtrsim$ $\left\|\mathbf{c}_{j}\right\|_{\ell_{2}}$ are valid, i.e., that $\Phi_{j}$ and $\tilde{\Phi}_{j}$ are uniform $L_{2}$ (I)-Riesz bases for their spans, it suffices to verify that $\left\langle\Phi_{j}, \tilde{\Phi}_{j}\right\rangle_{L_{2}(\mathrm{I})}$ is invertible, with an inverse that is bounded uniformly in $j$, which we will do below. Indeed, from this property and $\left\|\mathbf{c}_{j}^{\top} \Phi_{j}\right\|_{L_{2}(\mathrm{I})} \lesssim$ 


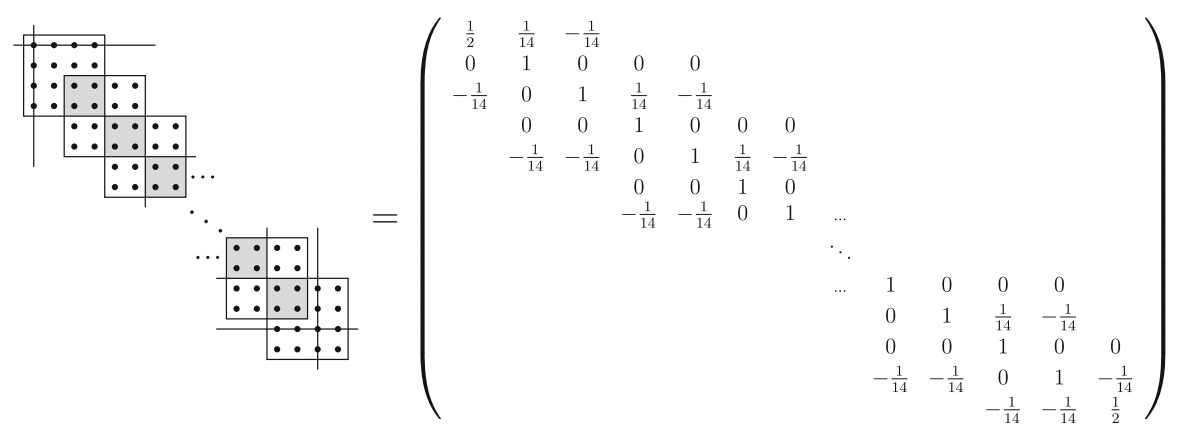

Fig. 7 The assembling of $\left\langle\Phi_{j}^{(1)}, \tilde{\Phi}_{j}^{(1)}\right\rangle_{L_{2}(\mathrm{I})}$ from the $4 \times 4$ left upper block from (11)

$\left\|\mathbf{c}_{j}\right\|_{\ell_{2}}$, we obtain

$$
\begin{aligned}
& \left\|\tilde{\mathbf{c}}_{j}\right\|_{\ell_{2}} \lesssim\left\|\left\langle\Phi_{j}, \tilde{\Phi}_{j}\right\rangle_{L_{2}(\mathrm{I})} \tilde{\mathbf{c}}_{j}\right\|_{\ell_{2}}=\sup _{\mathbf{c}_{j} \neq 0} \frac{\left|\left\langle\mathbf{c}_{j},\left\langle\Phi_{j}, \tilde{\Phi}_{j}\right\rangle_{L_{2}(\mathrm{I})} \tilde{\mathbf{c}}_{j}\right\rangle_{\ell_{2}}\right|}{\left\|\mathbf{c}_{j}\right\|_{\ell_{2}}} \\
& =\sup _{\mathbf{c}_{j} \neq 0} \frac{\left|\left\langle\mathbf{c}_{j}^{\top} \Phi_{j}, \tilde{\mathbf{c}}_{j}^{\top} \tilde{\Phi}_{j}\right\rangle_{L_{2}(\mathrm{I})}\right|}{\left\|\mathbf{c}_{j}\right\|_{\ell_{2}}} \lesssim\left\|\tilde{\mathbf{c}}_{j}^{\top} \tilde{\Phi}_{j}\right\|_{L_{2}(\mathrm{I})} \sup _{\mathbf{c}_{j} \neq 0} \frac{\left\|\mathbf{c}_{j}^{\top} \Phi_{j}\right\|_{L_{2}(\mathrm{I})}}{\left\|\mathbf{c}_{j}\right\|_{\ell_{2}}} \lesssim\left\|\tilde{\mathbf{c}}_{j}^{\top} \tilde{\Phi}_{j}\right\|_{L_{2}(\mathrm{I})},
\end{aligned}
$$

and similarly, using $\left\|\tilde{\mathbf{c}}_{j}^{\top} \tilde{\Phi}_{j}\right\|_{L_{2}(\mathrm{I})} \lesssim\left\|\tilde{\mathbf{c}}_{j}\right\|_{\ell_{2}}$, that $\left\|\mathbf{c}_{j}\right\|_{\ell_{2}} \lesssim\left\|\mathbf{c}_{j}^{\top} \Phi_{j}\right\|_{L_{2}(\mathrm{I})}$.

For $i \neq \imath^{\prime},\left\langle\Phi_{j}^{(i)}, \tilde{\Phi}_{j}^{\left(l^{\prime}\right)}\right\rangle_{L_{2}(\mathrm{I})}=0$, and $\left\langle\Phi_{j}^{(2)}, \tilde{\Phi}_{j}^{(2)}\right\rangle_{L_{2}(\mathrm{I})}$ is a diagonal matrix that is uniformly spectrally equivalent to the identity matrix. The matrix $\left\langle\Phi_{j}^{(1)}, \tilde{\Phi}_{j}^{(1)}\right\rangle_{L_{2}(\mathrm{I})}$ is assembled from the $4 \times 4$ left upper block from (16) as indicated in Fig. 7, where in the overlays the matrices should be added. The striking out of the first and the one but last rows and columns corresponds to the fact that for $k \in\left\{0,2^{j+2}\right\}, \phi_{j, k}^{(1)}$ and $\tilde{\phi}_{j, k}^{(1)}$ are not in $\Phi_{j}$ and $\tilde{\Phi}_{j}$, respectively. By multiplying the first and last rows and columns of $\left\langle\Phi_{j}^{(1)}, \tilde{\Phi}_{j}^{(1)}\right\rangle_{L_{2}(\mathrm{I})}$ with $\sqrt{2}$, a matrix of the form $I-B_{j}$ is obtained, where $\left\|B_{j}\right\| \leq \sqrt{\left\|B_{j}\right\|_{1}\left\|B_{j}\right\|_{\infty}} \leq \rho$ for some $\rho<1$ independent of $j$. Such a matrix is invertible, with a uniformly bounded inverse, with which the proof of Theorem 2 is completed.

Open Access This article is distributed under the terms of the Creative Commons Attribution Noncommercial License which permits any noncommercial use, distribution, and reproduction in any medium, provided the original author(s) and source are credited.

\section{References}

1. Bungartz, H., Griebel, M.: Sparse grids. Acta Numer. 13, 147-269 (2004)

2. Cohen, A.: Numerical Analysis of Wavelet Methods. Elsevier, Amsterdam (2003)

3. Cohen, A., Dahmen, W., DeVore, R.: Adaptive wavelet methods for elliptic operator equations-convergence rates. Math. Comput. 70, 27-75 (2001) 
4. Dahlke, S., Weinreich, I.: Wavelet-Galerkin methods: an adapted biorthogonal wavelet basis. Constr. Approx. 9(2-3), 237-262 (1993)

5. Dahmen, W.: Stability of multiscale transformations. J. Fourier Anal. Appl. 4, 341-362 (1996)

6. Dahmen, W.: Wavelet and multiscale methods for operator equations. Acta Numer. 6, 55-228 (1997)

7. Dahmen, W., Stevenson, R.: Element-by-element construction of wavelets satisfying stability and moment conditions. SIAM J. Numer. Anal. 37(1), 319-352 (1999)

8. Dauge, M., Stevenson, R.: Sparse tensor product wavelet approximation of singular functions. Technical report (2009, submitted)

9. Dijkema, T., Schwab, C., Stevenson, R.: An adaptive wavelet method for solving high-dimensional elliptic PDEs. Constr. Approx. 30(3), 423-455 (2009)

10. Gantumur, T., Harbrecht, H., Stevenson, R.: An optimal adaptive wavelet method without coarsening of the iterands. Math. Comput. 76, 615-629 (2007)

11. Lions, J.L., Magenes, E.: Non-homogeneous boundary value problems and applications, vol. I. Springer, New York (1972). Translated from the French by P. Kenneth, Die Grundlehren der mathematischen Wissenschaften, Band 181 